\title{
BOLETIN CRIMINOLÓCICO
}

No 84

Instituto andaluz interuniversitario de Criminología

Una vez más el Boletín Criminológic o ofrece a sus lectores un estudio sobre el tema de la comupción pública. En esta ocasión el presente trabajo tiene como principal objetivo el estudio de este fenómeno en México, a través del análisis de las representaciones sociales que sobre dicho problema tiene un sectorespecífico de la sociedad mexcana. En este estudio se recoge, en primer lugar, el marco teórico de la materia, describiendo las principales comientes que definen el fenómeno y explic an sus causas. En segundo lugar, se hace referencia a la situación mexicana en el contexto intemacional actual. Finalmente, se exponen los resultados obtenidos en una investigación empínca sobre las opiniones que al respecto tienen estudiantesy profesionales del ámbito juńdico en el Distrito Federal.

Palabras clave: comupción, representaciones sociales, administración pública.

\section{LA CORRUPCIÓN PÚBLICA EN MÉXICO: REPRESENTACIONES SOCIALES DE LA COMUNIDAD JURÍDICA DEL DISTRITO FEDERAL.}

\author{
José Cruz García González \\ Rosa Obdulia González Robles \\ Adolfo Mir Araujo \\ Arancha Garcia del Soto
}

\section{CONCEPTO Y CAUSAS}

La corrupción es una patología social tan antigua como las primeras civilizaciones. Ha estado presente en diversas formas e intensidades durante todas las épocas y en todas las culturas. Mucho antes de la aparición del Estado moderno, el Código de Hammurabi ya regulaba el comportamiento de los empleados públicos. Más de trece siglos después, en su estudio sobre la política, Aristóteles distinguió tres tipos de gobierno o regímenes políticos: la monarquía, la aristocracia y la república, así como tres desviaciones o corrupción de los mismos. En ese contexto señaló como desviación o corrupción de la monarquía a la tiranía, afirmando que "tanto la una como la otra son formas de gobierno concentradas en una sola persona, pero mientras que el tirano estudia su propia ventaja, el rey busca la de sus súbditos".

El debate sobre las posibles causas que han dado lugar al surgimiento y expansión de la corrupción pública ha sido igualmente intenso. Destacan las explicaciones estructurales, según las cuales la corrupción sería sólo una señal manifiesta de los defectos o desajustes de la organización social en sí: Sería consecuencia directa del resultado de los procesos de modernización, de las desigualdades económicas, de la pobreza y de las formas de dominación política. Otra corriente explicativa se basaría en la existencia de una serie de causas culturales que habrían propiciado su surgimiento. Por último, se podría resaltar aquella otra que centraría su exposición en la teoría de mercado o teorías económicas. Sin embargo, dependiendo de la gravedad y extensión con que se presente el problema en un país determinado es posible que más de una de estas corrientes al mismo tiempo permita ofrecer una explicación convincente.

\section{LA SITUACIÓN MEXICANA EN} EL CONTEXTO INTERNACIONAL

La ONG Transparencia Internacional entrega cada año el más completo y reconocido índice de percepción de la corrupción en el ámbito internacional. EL IPC de 2002 cubre 102 países, siendo percibidos como los menos corruptos aquellos que alcanzan puntuaciones cercanas a 10 . De los países de América Latina, México ocupa el puesto 57 del índice, al igual que Colombia, con 3.6 puntos, situándose por detrás de Chile (7.5), Uruguay (5.1), Costa Rica (4.5.), Perú (4.0) y Brasil (4.0), quienes obtuvieron las calificaciones más altas del continente americano. En octubre de 2001 la misma organización dio a conocer los resultados de una encuesta en la que se evaluó el nivel de corrupción de cada estado del país. De acuerdo a los resultados, la ciudad de México DF obtuvo la calificación más negativa, ya que, de cada 100 trámites burocráticos realizados por los ciudadanos, en 22.62 de los casos resultó indispensable dar una "mordida" o soborno a las autoridades responsables para obtener el servicio. Actualmente se estima que el país pierde un $9.5 \%$ del Producto Interno Bruto a causa de la corrupción y que, tan solo en sobornos, se gastan anualmente 23 mil millones de pesos, aproximadamente 2,300 millones de dólares americanos.

D irectora: A na I sabel Cerezo D omínguez. Coordinadora: Fátima Pérez Jiménez. Publicado por la Sección de M álaga del IAIC. Edificio Institutos de Investigación, U niversidad de M álaga. Campus deTeatinos, 29071 M A LAGA Tel: (95) 2132325 Fax: (95) 21322 42. D epósito legal: M A 857/ 1996. ISSN :1137-2427. www.uma.es/ estudios/propias/criminologia, boletincriminologico@ uma.es 


\section{METO DOLOG ÍA}

Se han analizado 294 cuestionarios en el periodo comprendido entre el 3 de diciembre de 2001 y el 15 de enero de 2002. La muestra estuvo compuesta por un grupo de funcionarios del poder judicial asignados a un juzgado de cada una de las tres prisiones del Distrito Federal; un grupo de funcionarios de dos agencias del ministerio públic o de la Proc uraduńa General de J usticia; un grupo de abogados litigantes; así como varios grupos de alumnos de la carrera de derecho de tres universidades de la Ciudad de México (una pública y dos privadas). Dado que la encuesta no se basó en una muestra totalmente aleatoria, sus resultados deberán tomarse en cuenta como sugerentes y no como representativos de la población entera. No obstante, los datos obtenidos aportan un indicador importante de las representaciones sociales que sobre el fenómeno tiene un sector relevante de la sociedad del Distrito Federal. Del total de sujetos encuestados el $23.8 \%$ comespondió a profesionales y el $76.2 \%$ a estudiantes de la camera de derecho; el $61.3 \%$ de la muestra trabajaba y la edad promedio de la muestra fue de 24 años, siendo de 31 años para los profesionales y de 21 para los estudiantes.

\section{REPRESENTACIONES SOCIA- LES DE LA CORRUPCIÓN PÚ- BLICA EN MÉXICO}

Siempre que se analiza un fenómeno social como el de la corrupción pública resulta interesante conocer las actitudes y concepción que sobre el tema tienen determinados sectores de la sociedad. En este caso, se pretendió conocer las representaciones que del fenómeno tienen diversos profesionales del mundo del derecho y estudiantes del Distrito Federal. La comunidad jurídica por su naturaleza tiene un contacto muy intenso con los diversos sectores de la administración pública de cualquier país, lo que le permite formarse representaciones fundadas en la práctica del funcionamiento de la administración pública de la sociedad en que se desenvuelven. Para este estudio se escogió la comunidad de Ciudad de México atendiendo a que dicha ciudad obtuvo el índice más alto de corrupción en el país de acuerdo a la encuesta de Transparencia Mexicana, tal como se ha mostrado supra.

\section{RESULTADOS}

A la muestra de la comunidad jurídica del Distrito Federal se le pidió en una de las preguntas que asignase un valor que iba de un mí- nimo de 1 (muy leve) a un máximo de 5 (muy grave) a una serie de problemas del Distrito Federal que se recogen en la tabla 1. De estos problemas los que obtuvieron las medias más elevadas fueron la inseguridad ciudadana y la delincuencia (4.63), el desempleo (4.36) y la corrupción pública (4.31). La tabla 1 muestra los porcentajes de calificación de grave y muy grave que dieron los encuestados que trabajan en el sector público, en el privado o que son exclusivamente estudiantes (clasificados en "otro"), a estos diferentes problemas. Como se observa en dicha tabla, quienes desempeñan sus actividades

\begin{tabular}{|l|c|c|c|c|}
\hline \multirow{2}{*}{ Tabla 1 P R O B L E M A } & \multicolumn{3}{c|}{ \% acumulado niveles grave/muy grave } \\
\hline & Público & Privado & Otro & Total \\
\hline Coste de vida & 70.5 & 68.30 & 56.9 & 64.3 \\
\hline Tráfico-consumo de drogas & 76.70 & 67.10 & 70.7 & 71.6 \\
\hline Recesión económica & 73.00 & 77.60 & 77.2 & 76.0 \\
\hline Desempleo & 90.0 & 85.0 & 82.5 & 85.5 \\
\hline Corrupción pública & 77.60 & 87.60 & 83.9 & 82.9 \\
\hline Procuración de justicia & 50.60 & 79.70 & 68.7 & 66.1 \\
\hline Impartición de justicia & 43.80 & 75.10 & 73.5 & 64.5 \\
\hline Inseguridad pública y delincuencia & 94.40 & 93.80 & 87.9 & 91.6 \\
\hline Coste de la educación & 52.30 & 64.60 & 56.3 & 57.3 \\
\hline Coste de los servicios de salud & 52.80 & 60.30 & 58.9 & 57.3 \\
\hline Fuerzas policiales & 72.70 & 76.00 & 73.6 & 74.0 \\
\hline Corrupción del sector privado & 36.00 & 39.80 & 58.3 & 45.8 \\
\hline La falta de vivienda & 59.50 & 64.6 & 68.7 & 64.7 \\
\hline
\end{tabular}

en el sector privado o son exclusivamente estudiantes tienden a atribuir una mayor gravedad al problema de la corrupción pública que los que trabajan en el sector público.

Una vez conocido el nivel de preocupación de la comunidad jurídica ante el problema de la corrupción pública, similar al que ocasiona la recesión económica y sólo por debajo del desempleo y la inseguridad ciudadana y la delincuencia, consideramos conveniente conocer, a través de la siguiente pregunta, los mecanismos de operación de la corrupción.
Es por ello que se les preguntó acerca de cómo consideraban que se consumaban los actos de corrupción en los que intervenían miembros de la comunidad jurídica. Tres posibles mecanismos fueron considerados en el estudio, al preguntar si el funcionario es quien solicita el pago de un soborno, éste es ofrecido por el abogado o bien ya es sabido cuánto hay que pagar. Que el funcionario solicite el pago supone que el acto de corrupción es resultado de una decisión que toma el funcionario, bajo su propio riesgo, aprovechando el poder de que dispone para dispensar beneficios o imponer perjuicios en virtud del cargo que desempeña. Que sea el abogado quien tome la iniciativa supone que, también bajo su riesgo, se sirva de la oferta de soborno para inducir a un funcionario a incumplir los deberes de su cargo. Saber cuánto hay que pagar supone que las prácticas corruptas constituyen el modo habitual de funcionamiento de la administración pública y que no es necesario llevar a cabo una invitación explícita a incurrir en una conducta irregular Constituye lo que podría denominarse 
Tabla 2

\begin{tabular}{|l|c|c|c|}
\hline \multirow{2}{*}{ Un abogado paga soborno porque... } & \multicolumn{2}{|c|}{ Estatus } & \multirow{2}{*}{ Total } \\
\cline { 1 - 4 } & Profesional & Estudiante & \\
\hline El funcionario público solicita el pago & $13.2 \%$ & $11.4 \%$ & $11.8 \%$ \\
\hline El abogado ofrece el pago & $60.3 \%$ & $12.7 \%$ & $24.0 \%$ \\
\hline Ya se sabe cuánto hay que pagar & $26.5 \%$ & $75.9 \%$ & $64.2 \%$ \\
\hline
\end{tabular}

la institucionalización de la corrupción, en la que el comportamiento corrupto adquiere las notas de máxima impersonalidad y ausencia de reconocimiento de responsabilidad personal.Como puede observarse en la tabla 2, la gran mayoría de los encuestados considera que el mecanismo de operación de la corrupción consiste en procedimientos ya establecidos, que son conocidos y observados al igual por funcionarios y por abogados. En tanto que las dos terceras partes de los encuestados contestan que "se sabe cuánto hay que pagar", es reducido el porcentaje de quienes declaran que el abogado ofrece el pago y aún menor el de quienes atribuyen al funcionario solicitarlo. Debe repararse, sin embargo, en que en buena medida estos resultados se deben a la composición de la muestra, de la que el $76.2 \%$ son estudiantes. Profesionales y estudiantes responden de un modo totalmente diferenciado. Mientras el $60 \%$ de los profesionales achaca al abogado el inducir al sobor- mismo modo en el público. Si relacionamos entre si las tablas 2 y 3 , observamos que muestran un escenario claramente acorde con el enfoque económico del fenómeno de la corrupción. Si la práctica del soborno llega a ser un procedimiento habitual dentro de la administración pública, ello puede ser debido al elevado grado de impunidad. Donde el riesgo de castigo es bajo, el costo de la corrupción es también bajo para el funcionario y el beneficio comparativamente alto. Para el abogado, negarse a sobornar significa un costo muy alto, ya que prácticamente implica ser in-
Tabla 3

che personal.

Esta representación del carácter de la corrupción es consistente con el hecho de que casi el $60 \%$ de los encuestados declare que fue emplazado, en los últimos dos años, al pago de soborno para obtener algún servicio o resolver un problema que tuviera que ver con alguna dependencia de la administración pública. También encuentra apoyo esta idea en el hecho de que cerca del $75 \%$ de la muestra haya sido testigo, directo o indirecto de actos de corrupción relacionados con la administración pública durante los últimos dos años. Como muestra la tabla 4 , apenas el $6.5 \%$ de quienes se encontraron en esa situación declaró haber denunciado el hecho.

Como se aprecia en la tabla 5 , las razones para no denunciar que obtienen los porcentajes más elevados expresan más bien una actitud de pesimismo y resignación ante el con-

\begin{tabular}{|c|r|r|r|}
\hline \multirow{2}{*}{ Probabilidad de éxito del soborno } & \multicolumn{2}{|c|}{ Sector de empleo } & \multirow{2}{*}{ Total } \\
\cline { 2 - 4 } & Público & Privado & \\
\hline Muy Baja & $16.9 \%$ & & $8.9 \%$ \\
\hline Baja & $9.0 \%$ & & $4.7 \%$ \\
\hline Media & $25.8 \%$ & $12.5 \%$ & $19.5 \%$ \\
\hline Alta & $27.0 \%$ & $36.3 \%$ & $31.4 \%$ \\
\hline Muy Alta & $21.3 \%$ & $51.3 \%$ & $35.5 \%$ \\
\hline
\end{tabular}

Tabla 4

\begin{tabular}{|l|c|c|c|}
\hline \multicolumn{1}{|c|}{$\begin{array}{c}\text { El acto de corrupción del } \\
\text { que fué testigo: }\end{array}$} & \multicolumn{2}{c|}{ Estatus } & \multirow{2}{*}{ Total } \\
\cline { 1 - 4 } & Profesional & Estudiante & \\
\hline Lo denunció & $2.2 \%$ & $7.6 \%$ & $6.5 \%$ \\
\hline No lo denunció & $97.8 \%$ & $92.4 \%$ & $93.5 \%$ \\
\hline
\end{tabular}

no, el $75 \%$ de los estudiantes piensa que el mecanismo de la corrupción ya está montado y precede a las acciones de funcionarios y abogados. Curiosamente, sólo coinciden en excluir que sea el funcionario quien inicie el acto de soborno.

Si dirigimos la atención a la tabla 3 encontramos que dos terceras partes de la muestra perciben al soborno como un medio muy eficaz para lograr un servicio eficiente por parte de la administración, al asignarle una probabilidad alta o muy alta de éxito. Aquí la diferencia es muy apreciable por sector de empleo. Aunque en ambos sectores se le reconoce como un medio muy efectivo, en el privado casi el $90 \%$ de los encuestados le concede una alta o muy alta probabilidad de éxito, mientras menos de la mitad lo califica del competente a los ojos de su clientela. Ante tal situación, apenas cabe duda de cuál será su comportamiento, dado que la habitualidad de la situación difícilmente conducirá ni a reprensión profesional o social ni al autorrepro-

\section{Tabla 5}

\begin{tabular}{|l|c|}
\hline \multicolumn{1}{|c|}{$\begin{array}{c}\text { Los casos de corrupción no se denuncian } \\
\text { porque: }\end{array}$} & $\begin{array}{c}\text { Importante o } \\
\text { Muy Importante }\end{array}$ \\
\hline Los procedimientos son demasiado complejos & $50.0 \%$ \\
\hline Nadie puede probar nada & $50.0 \%$ \\
\hline Los instrumentos legales son ineficaces & $60.6 \%$ \\
\hline Éstos no se castigan ni se investigan & $67.4 \%$ \\
\hline La situación económica los justifica & $41.3 \%$ \\
\hline Son aceptados culturalmente en México & $47.2 \%$ \\
\hline No existe protección ante posibles represalias & $76.6 \%$ \\
\hline Son tan triviales que no vale la pena & $32.6 \%$ \\
\hline Los denunciados logran evadir responsabilidad & $73.1 \%$ \\
\hline
\end{tabular}

vencimiento de que la ciudadanía es impotente frente al andamiaje montado por el mismo sistema político para asegurar la impunidad de quienes desempeñan funciones públicas. El sentimiento de indefensión del ciudadano ante el poder público se manifiesta en que el temor a las represalias es el motivo más importante por el que no se denuncian los casos de corrupción Los datos de esta tabla nos indican cómo la percepción del 
fenómeno de la corrupción está condicionada por los rasgos estructurales del sistema político mexicano. Si por un lado los mexicanos han aprendido a aceptar la corrupción, en parte porque la reconocen como una fuente de beneficios de los que, en las circunstancias propicias, pueden beneficiarse, por otro lado tienen una clara conciencia de que el sistema que la propicia tiene rasgos autoritarios.

\section{ESTRATEGIAS CONTRA LA CORRUPCIÓN}

Durante los últimos años el tema de la prevención y la reducción de la corrupción pública han sido vistos principalmente desde la perspectiva legal o institucional, siendo este acercamiento claramente necesario en la lucha contra la corrupción. Sin embargo, durante los próximos años este enfoque seguramente será insuficiente si junto a él no se establece una estrategia complementaria que incluya un cambio fundamental en el sistema de valores de la sociedad mexicana, para que las medidas adoptadas sean acompañadas por una sociedad cada vez más afín a una cultura de legalidad. En ese sentido, podemos señalar cuatro principales estrategias contra la corrupción:

\section{Sancionar las transgresiones graves}

Cuando hay una cultura de impunidad generalizada, la única manera de romperla es que personalidades corruptas importantes sean declaradas culpables y castigadas. Es decir, para combatir la generalización de una cultura de la impunidad, es necesario generalizar una cultura de legalidad. Para ello se considera indispensable una seria revisión de la legislación penal en la materia, con el objeto de incrementar al máximo posible los riesgos para aquellos que decidan desviarse de las funciones naturales del cargo público para atender intereses privados mediante la obtención de cualquier beneficio indebido.

\section{Involucrar al pueblo en el diagnóstico de los sistemas corruptos}

Las campañas exitosas involucran al pueblo. Si se logra incentivar una cultura de legalidad, los ciudadanos son fuentes fértiles de información acerca de dónde ocurre la corrupción. Los mecanismos para consultarlos incluyen encuestas sistemáticas, organismos ciudadanos de vigilancia de las agencias públicas, participación de organizaciones profesionales, líneas telefónicas directas, programas educativos, consejos de aldea y barrio, etc.

\section{Concentrarse en la prevención mediante la reparación de los sistemas corruptos}

Metafóricamente la corrupción puede ser caracterizada por la siguiente fórmula o "ecuación básica": "Corrupción = Monopolio + Discrecionalidad - Responsabilidad".

En ese sentido, el combate contra la corrupción pública en México o en cualquier otro país debe empezar con mejoras en el sistema de funcio- namiento del sector público, acompañadas de "evaluaciones de vulnerabilidad". Por ello, antes que formar funcionarios y ciudadanos incorruptibles, es imprescindible promover la competencia, cambiar incentivos y mejorar la rendicn de cuentas en el sector público; en pocas palabras, corregir los sistemas que engendran corrupción.

\section{Incentivos de reforma}

Los gobiernos que desean detener la corrupción deben mejorar los incentivos. Cuando las remuneraciones del sector público han caído tan bajo que una familia no puede mantenerse con el salario de un funcionario típico, no debe sorprender que prospere la corrupción.

\section{Fortalecer la cooperación internacional}

Considerando el contexto internacional actual y el hecho de que en muchos casos los funcionarios públicos corruptos huyen de sus países junto con los bienes producto de la corrupción, ya sea a paraísos fiscales ya sea a otros países donde no existan tratados de extradición con el de su procedencia, una estrategia contra la corrupción será siempre incompleta si no se fortalece la cooperación internacional en la materia. Por ello, es vital que países como México se integre en todas las iniciativas internacionales posibles, facilitando así el intercambio de información y la persecución de estos delitos a través de las fronteras.

\section{CONGRESO ESPAÑOL DE CRIMINOLOGÍA}

Los días 5,6 y 7 de abril de 2006 tendrá lugaren la Facultad de Derecho de la UNED en Madrid el III Congreso español de Criminología. Organizan la Sociedad Española de Investigación Criminológica (SEIC) y la Federación de asociaciones de criminólogos de España (FACE). Más información en la web:

http://uww.c riminologia,net

\section{CONGRESO PENITENCIARIO INTERNACIONAL BARC ELONA 2006}

Los días 30 y 31 de marzo y 1 de abril de 2006 tendrá lugaren Barcelona el Congreso Penitenciario Intemacional bajo el tútulo

"La función social de la política penitenciaria".

Dicho encuentro contará con la participación de los más destacados expertos intemacionales en los ámbitos de la criminología, la política criminal y la gestión penitenciaria. El Congreso está organizado conjuntamente por la autoridades penitenciarias de los gobiemos central de España y autónomo de Cataluña y cuenta con la colaboración del Consejo de Europa y de su Consejo Cientíico Criminológico.

Las lenguas de trabajo serán: castellano, catalán, francése inglés. Se dispondrá de un servicio de traducción simultánea.

La inscripción al congreso ya está abierta. Para más información: 